\title{
PRESENTACIÓN DE LA SECCIÓN DOSIER LAS NUEVAS FORMAS DE COMUNICACIÓN EDUCATIVA: AULA EXTENDIDA
}

doi: https://doi.org/10.26439/contratexto2021.n036.5559

La edición 36 de Contratexto fue propuesta en el año 2019, mucho antes de la aparición en la escena mundial de la pandemia por el COVID-19 que todavía se encuentra entre nosotros. Entonces, nos propusimos abordar las nuevas formas de comunicación educativa enfocadas en el aula extendida. Esta fue concebida por Barberà y Badia (2004) como una propuesta pedagógico-tecnológica basada en el dictado de un curso de modalidad prácticamente presencial combinada con el aula aumentada con tecnologías digitales (TD), en la cual se aprovechan diversos soportes tecnológicos que permiten extender la acción docente dentro de la propuesta de formación, más allá de los medios tradicionales de la propia clase. Ahora bien, al colocar la mirada en la realidad en la cual nos encontramos inmersos, se han develado nuevos indicadores definitorios de este concepto, sentidos y lenguajes para definir el aula extendida, desde los caminos para emitir mensajes, el empleo de diversos recursos, los medios o estrategias aplicadas en los procesos de enseñanza y aprendizaje que superan las barreras del enfoque físico del aula.

De igual modo, los autores en este dosier han dejado planteados los cimientos o pesquisas sobre las cuales gira en la actualidad el divergente contexto educativo, que ha abierto posibilidades que anteriormente eran pensadas solo para el espacio del aula. Esto implica, por lo tanto, reflexionar sobre el desarrollo del pensamiento disruptivo, crítico, creativo, y la equidad o real eficacia de los recursos mediatizados como el aprendizaje móvil (mobile-learning), aprendizaje con tablets (tablet-learning), plataformas educativas, las nuevas percepciones de las tecnologías para aprender y conocer (TAC), emprender y participar (TEP).

En este número, Contratexto reconoce que, ante las nuevas realidades planteadas en los contextos previamente enunciados, los docentes han emprendido nuevas formas de socialización que giran alrededor del fortalecimiento de las habilidades sociales, como el trabajo en equipo; se resalta a partir del mes de marzo del 2020 el rol del docente y su liderazgo para generar una comunicación e interacción dentro del enfoque del aula extendida.

En virtud de ello, esta edición presenta investigaciones y experiencias recientes surgidas durante la pandemia. Se trata de buenas prácticas que contemplan nuevas 
narrativas, una nueva semántica y nuevas metodologías que se tejen en el marco una nueva didáctica multidiversa que otorga sentido a la sociedad del conocimiento y al vínculo en comunicación y educación que se vive en todos los niveles curriculares. Al mismo tiempo, los artículos permiten observar una transformación en la enseñanza de las ciencias sociales, ciencias naturales, tecnología, matemáticas e innovación en los países iberoamericanos. De allí que se ofrecen trabajos provenientes de Argentina, Colombia, Perú y México.

Este número 36 de Contratexto coloca en el tapete los planteamientos del siglo XXI en cuanto a las nuevas formas de comunicación educativa asociadas al conectivismo y socioconstructivismo, entre otras tendencias teóricas que las sustentan.

Además, revela el significado de la transformación social que genera el acceso a los nuevos medios y la oportunidad para la participación ciudadana activa desde la escuela; y promueve determinadas habilidades sociales que exigen los divergentes escenarios que ha traído consigo el año 2020 y que continúan en el 2021 con la presencia entre nosotros de la pandemia del COVID-19. No obstante, no nos quedamos con aquello que en apariencia se visualiza, sino que nos hemos planteado descubrir los hilos invisibles del aprendizaje. Dutton (citado por Cobo y Moravec, 2011, p. 19) afirma que es hora de que los estudiantes, las madres, los padres y los educadores adopten una visión más amplia de internet en el aprendizaje y la educación. Es fuera de las aulas donde el potencial puede ser más significativo, donde la comprensión de estas dinámicas podría mejorar las iniciativas planeadas en las aulas u otros espacios de la educación formal.

Este dosier se interesa en los hilos que se tejen en la formación mediada por las tecnologías; el uso combinado de recursos (con los medios tradicionales) para promover entornos de aprendizaje más allá del aula; el trabajo y apoyo entre pares; el crecimiento de las comunidades participativas; la producción de contenidos educativos generados por usuarios en distintas plataformas; las barreras y oportunidades para el desarrollo del pensamiento crítico, así como debates teóricos y epistemológicos pertinentes a estos temas.

Comenzamos con el artículo denominado "Universidad y TIC: estudio de caso de una experiencia educativa en Salta, Argentina, en contexto de pandemia", escrito por María Rosa Chachagua y Silvana Aixa Hnilitze, que nos aproxima a la realidad vivida en Argentina desde marzo del 2020 por la suspensión de las clases presenciales en todo el país a raíz de la pandemia causada por el COVID-19. Específicamente nos muestran un estudio de caso combinado con una sistematización de experiencia del aula extendida de una cátedra anual de la Universidad Nacional de Salta en el periodo previo a la pandemia, donde se utilizaban algunas herramientas como Classroom, un blog y una página de Facebook; y posteriormente el uso de un aula virtual en la plataforma Moodle. El estudio concluye que el aula extendida es la opción más viable para el retorno a la presencialidad en un contexto próximo (pospandemia). 
Luego se presenta el artículo "Disciplina, gubernamentalidad y virtualidad en el aula extendida: notas de 'Aprende en Casa', la estrategia educativa mexicana ante la pandemia del COVID-19", escrito por Omar Daniel Cangas. En este ensayo se sustenta el sentido de algunas percepciones de estudiantes y docentes críticos, de lo que fue (y es aún) ejercer su práctica profesional y vivir los procesos formativos desde la estrategia "Aprende en Casa", y además de la sensación, quizás compartida, de haber perdido el espacio esencial para el logro de los aprendizajes: la escuela y sus aulas.

El trabajo "El uso del smartphone para la gestión del trabajo colaborativo en estudiantes de educación superior argentina durante la pandemia del COVID-19", escrito por Francisco Javier Albarello, Francisco Hernando Arri y Ana Laura García Luna, retrata en sus líneas cómo la situación de aislamiento social obligatorio debido a la pandemia global del COVID-19 ha afectado a todos los órdenes de la vida, y la educación no ha sido la excepción. El artículo presenta los resultados de una investigación cualitativa que incluyó entrevistas en profundidad a 45 estudiantes universitarios argentinos, con el objeto de indagar sobre los cambios que ha generado la cuarentena en las formas de estudio. Los hallazgos indican que los estudiantes desarrollan complejas estrategias de estudio colaborativo en las que alternan entre textos, audios y otros materiales y aplicaciones que les permiten estudiar y compensar la ausencia física de sus compañeros a través del trabajo colaborativo mediado por las pantallas.

Posteriormente, el estudio titulado "Development of Soft Skills from Out-of-School Experiences in Peru. Learnings from Non-Profit Organizations' Activities in Public Schools in Lima", escrito por Carla Lizette Mendoza Egúsquiza, tuvo como objetivo examinar la mejora de las habilidades sociales (HH. SS.) en la educación pública en el Perú a través del análisis de las técnicas de las organizaciones sin ánimo de lucro (OSAL) en el ámbito escolar. Los hallazgos de este escrito incluyen iniciativas gubernamentales que indirectamente desarrollan HH. SS., pero también evidencian problemas de gestión por una metodología que prioriza a los profesores en lugar de los estudiantes, lo que desalienta este desarrollo. Las áreas vulnerables suelen ser entornos hostiles para el desarrollo de habilidades, transformando las OSAL en espacios disruptivos complementarios para cultivar valores, ética y habilidades. El análisis del discurso concluye que, a pesar del corto alcance, principalmente desarrollan HH. SS. relacionadas con la colaboración y el desempeño de tareas, lo que impacta en las decisiones vocacionales, en las familias y comunidades. Las OSAL emplean una metodología lúdica y concreta que fomenta el desarrollo de HH. SS., pero difícil de implementar en escuelas públicas, debido a la falta de gestión y recursos. Este estudio es pionero en la región.

El artículo "La radio y la televisión en 'Aprendo en casa': hallazgos por codificación abierta", escrito por Luis-Rolando Alarcón-Llontop, Karl Torres-Mirez, Sindy PasaperaRamírez y Mirtha Carrasco-Yovera, presenta una investigación sobre lo que significó la estrategia "Aprendo en casa", en Perú, en el año 2020, desde dos de sus recursos 
principales: la radio y la televisión. El objetivo fue analizar comunicacionalmente y evaluar los programas de ambos medios. Se discutió sobre 32 piezas de todos los niveles educativos en un periodo de siete meses, con 27 expertos comunicadores; y se evaluó la experiencia a partir de 50 actores-destino de la estrategia. El estudio fue metodológicamente naturalista, de enfoque cualitativo, tipologías hermenéuticas indirectas y fenomenológicas, y con diseño de codificación abierta. Los autores presentan ocho categorías generadas a pleno albedrío que arrojan resultados sobre sesgados contenidos y cuestionables formas de presentación; se visibilizan brechas en lo educativo, digital y generacional, así como desarticulación entre los ejes educación y comunicación. Lo encontrado aspira a contribuir a la mejora del diseño y al uso de recursos mediáticos a favor de la incursión educativa analizada y similar.

Desde Colombia, Andrés Lombana-Bermudez escribe "El blog colectivo como portal de un espacio de afinidad: desarrollando el aula híbrida en una clase universitaria en Colombia", un estudio de caso sobre una experiencia de aula híbrida desarrollada en una clase universitaria de Comunicación Social en ese país, en la que se construyó un espacio de afinidad por medio del uso de tecnologías de información y comunicación (TIC). Utiliza las teorías de espacio de afinidad, de James Paul Gee (2017), y de aprendizaje conectado de Mizuko Ito y otros (2013), y se basa en datos cualitativos recolectados durante cuatro interacciones de la asignatura Comunicación e Interactividad entre los años 2019 y 2021. Los resultados del estudio confirman que el blog colectivo es una herramienta útil para construir espacios de afinidad, promover el aprendizaje conectado y desarrollar aulas híbridas.

Finalmente, el artículo "Juegos transmedia 'Yo amo leer': experiencias inmersivas, espacios de afinidad, aprendizaje conectado y creación de vínculo con la comunidad", de Nohemí Lugo Rodríguez, María Elena Melón Jareda y Zaira Delia Rodríguez Pérez, explora las experiencias y los aprendizajes de estudiantes universitarios al participar en diferentes roles (jugadores, actores y staff) en juegos transmedia. Este caso de estudio se llevó a cabo en la universidad y se analiza nueve de los once juegos "Yo amo leer" realizados del 2014 al 2019 para fomentar la lectura. La participación en dichos eventos ha sido aproximadamente de 1048 estudiantes universitarios. Los resultados y hallazgos indican que estos juegos son experiencias inmersivas que generan disposición a la lectura y aprendizajes diversos, despiertan interés y proporcionan beneficios a la comunidad de la universidad. Se concluye que generan un espacio de afinidad intramuros y constituyen una estrategia efectiva para promover el aprendizaje conectado.

Al cierre de la edición del dosier número 36, entendemos que la virtualidad no es en sí misma la solución a todos los problemas que enfrenta la humanidad, y mucho menos con la brecha digital que existe. De igual modo, ha quedado en evidencia que medios de comunicación tradicionales como la prensa, la radio y la televisión han servido como mediadores en tiempos de distanciamiento social, al igual que las aplicaciones como WhatsApp, Facebook y el uso de plataformas para conferencias como Zoom, lo que nos 
indica que la comunicación, aparte de extendida, es híbrida. Por otra parte, los escritos que usted encontrará en este dosier confirman la esencia de la educación como el fenómeno que permite el progreso del mundo. Durante estos meses, y de seguro los que vienen, tanto docentes, directivos, estudiantes y padres decidieron no quedarse atrás, vencer adversidades, continuar con los procesos de enseñanza y aprendizaje más allá del espacio físico de la escuela. La educación abraza esperanzas, edifica sobre sueños y construye las generaciones que dirigirán el futuro de nuestras naciones.

Dra. Franahid D'silva Editora invitada Universidad Nacional Abierta, Venezuela

Mg. Mariana Gabriela Torres Editora invitada Universidad Nacional de la Patagonia Austral, Universidad Nacional de la Patagonia San Juan Bosco, Argentina

\section{REFERENCIAS}

Barberà, E., y Badia, A. (2004). Educar con aulas virtuales. Orientaciones para la innovación en el proceso de enseñanza y aprendizaje. Machado Libros.

Cobo, C., y Moravec, J. W. (2011). Aprendizaje invisible. Hacia una nueva ecología de la educación. Universidad de Barcelona.

Ito, M., Gutiérrez, K., Livingstone, S., Penuel, B., Rhodes, J., Salen, K., Schor, J., SeftonGreen, J., y Watkins, C. S. (2013). Connected Learning: An Agenda for Research and Design. Digital Media and Learning Research Hub. 